\title{
Calidad de vida y condiciones de salud de la población adulta mayor de Medellín
}

\author{
Doris Cardona ${ }^{1}$, Alejandro Estrada ${ }^{2}$, Héctor Byron Agudelo ${ }^{1}$ \\ ${ }^{1}$ Facultad Nacional de Salud Pública, Universidad de Antioquia, Medellín, Colombia. \\ ${ }^{2}$ Universidad de Antioquia, Escuela de Nutrición y Dietética, Medellín, Colombia.
}

Introducción. El proceso de envejecimiento presenta limitaciones funcionales, pérdida de autonomía y de independencia, de adaptabilidad y disfuncionalidad motriz, las cuales plantean retos y preocupaciones de vital importancia tanto para quienes las viven como para los organismos gubernamentales y que llevan a preguntarse cuáles son las condiciones de vida y de salud de la población adulta mayor.

Objetivo. Caracterizar algunos componentes de la calidad de vida: económicos, familiares, socio-demográficos, de seguridad social y de condiciones de salud de la población adulta mayor (de 65 años o más) en la ciudad de Medellín.

Materiales y métodos. Se realizó un estudio descriptivo con base en la información proveniente de la Encuesta de Calidad de Vida realizada en la ciudad en los años 1997 y 2001; los certificados de defunción de 1990 a 1999; los registros de egresos hospitalarios de 1990 a 1997, y una encuesta telefónica en el año 2002 a 637 adultos mayores seleccionados sistemáticamente.

Resultados. Se observó un incremento de 168\% en este grupo poblacional desde 1964 a 2001, evidenciando la etapa de transición demográfica plena que vive la ciudad. También se observó una pérdida de poder adquisitivo de 65\% entre 1997 y 2001, así como la situación de aislamiento en que viven los ancianos, lo que marca la pérdida de independencia y autoestima. Conclusión. Los componentes de independencia, seguridad socioeconómica y redes de apoyo social constituyen el $97 \%$ de la calidad de vida del adulto mayor de la ciudad.

Palabras clave: envejecimiento de la población, calidad de vida, apoyo social, seguridad social, condiciones de salud, Colombia.

\section{Quality of life and health conditions of the elderly population of Medellin}

Introduction. The process of aging presents functional limitations, loss of autonomy, independence, adaptability and motor disfunction. These changes raise challenges and concerns of vital importance both for those individuals who face them and for government organizations, thus motivating inquiry about the life and health conditions of the elderly.

Objective. To describe some components of the quality of life of the elderly population (aged 65 years or older) living in Medellin, including socio-demographic, family, social security and health conditions.

Materials and methods. A descriptive study was conducted using the following sources of information: a survey of quality of life that was carried out in 1997 and 2001; death certificates from 1990 to 1999; hospital discharge records from 1990 to 1997; and a telephone survey carried out in the year 2002 in 637 randomly selected elderly individuals.

Results. A $168 \%$ increase occurred in this population group from 1964 to 2001 , substantiating the stage of demographic transition throughout the city. The $65 \%$ loss of purchasing power observed between 1997 to 2001 and the situation of isolation in which the elderly population lives, characterize its loss of independence and self-esteem.

Conclusion. $97 \%$ of the quality of life in the elderly population of Medellin was explained by the following components: independence, social and economic security, and networks of social support.

Keywords: demographic aging, quality of life, social support, social security, health conditions, Colombia. 
En una época en que el descenso acelerado de la fecundidad modifica radicalmente el ritmo de crecimiento de la población y su estructura por edad, dos hechos llaman la atención: las personas viven en promedio más años que antes y se observa un importante crecimiento en el número de personas en edades avanzadas. Estos dos aspectos constituyen conceptos diferentes aunque relacionados: el primero es la prolongación de la vida de los individuos; el segundo corresponde al envejecimiento de las poblaciones, generalmente expresado en un aumento en la proporción de personas mayores, con repercusiones considerables en el funcionamiento de las estructuras sociales, en los sistemas de pensiones, jubilaciones, distribución de tareas dentro de las familias y un aumento en la necesidad de asistencia médica, lo que plantea retos importantes al sistema de salud (1).

El proceso de envejecimiento que presenta limitaciones funcionales, pérdida de autonomía, independencia y adaptabilidad y disfuncionalidad motriz es la consecuencia de dos transiciones: una es la demográfica-tendencias de la mortalidad y la fecundidad en el transcurso del tiempo- que se vive en los países de América y del mundo, lo cual genera demandas específicas y complejas de servicios y bienes sociales y de salud; la otra es la polarización epidemiológica, entendida como el proceso de repercusiones simultáneas y sustanciales sobre el perfil de mortalidad de la población, tanto por las enfermedades transmisibles como las no transmisibles, y las causas externas, especialmente en grupos vulnerables como los pobres y los ancianos (2), lo que origina un incremento de la población y con ello un cambio en la estructura por edad, lo cual a su vez genera profundas variaciones en la manera de ser y de pensar de las personas y de las instituciones, puesto que deben adaptarse a nuevos ritmos de vida social, aumento de los problemas, no sólo por el número creciente de individuos que llegan

\footnotetext{
Correspondencia:

Doris Cardona, Facultad Nacional de Salud Pública, Universidad de Antioquia, Calle 62 No. 52-59. Tel. 2106825, fax 511-2506, Medellín - Colombia.

doriscar @guajiros.udea.edu.co.

Recibido: 23/09/05; aceptado: 17/03/06
}

a la vejez sino por las dificultades institucionales para dar respuestas satisfactorias a sus necesidades, sino por las demandas en el ámbito económico, social, cultural, político y de salud.

También se deben considerar las diferencias derivadas del sexo: mientras el hombre adulto mayor de hoy fue el trabajador productivo de hace 20 años, el cual muere a una edad más temprana, la mujer tradicionalmente ha participado mucho menos en la fuerza laboral, tiene un acceso limitado a la obtención de ingresos y servicios esenciales, vive más tiempo, y es quien en la vejez, sobre todo las viudas sin apoyo familiar, corren el mayor riesgo de sufrir el deterioro de su calidad de vida (3).

La calidad de vida es una definición imprecisa y la mayoría de investigaciones que han trabajado en la construcción del concepto están de acuerdo en que no existe una teoría única que defina y explique el concepto. El término calidad de vida pertenece a un universo ideológico, no tiene sentido si no es en relación con un sistema de valores, y los términos que le han precedido en su genealogía ideológica remiten a una evaluación de la experiencia que de su propia vida tienen los sujetos; tal evaluación no es un acto de razón, sino más bien un sentimiento. Lo que mejor designa la calidad de vida es la calidad de la vivencia que de la vida tienen los sujetos (4).

A medida que aumentan la edad, la calidad de vida está determinada por la capacidad para mantener la autonomía,o sea, la capacidad percibida de controlar, afrontar y tomar decisiones personales acerca de cómo vivir al día de acuerdo con las normas y preferencias propias, y la independencia, es decir, la capacidad de desempeñar las funciones relacionadas con la vida diaria, en otras palabras, capacidad de vivir recibiendo poca o ninguna ayuda de los demás (5).

La Organización Mundial de la Salud ha definido la calidad de vida como la percepción individual de la propia posición en la vida dentro del contexto del sistema cultural y de valores en que se vive y en relación con sus objetivos, esperanzas, normas y preocupaciones (5). El concepto se construyó teniendo en cuenta la recomendación de la OMS de incluir aspectos físicos, psicológicos, 
sociales y ambientales (6) en las investigaciones que aborden esta temática; por esta razón, se entendió la calidad de vida como un fenómeno social complejo y un proceso de percepción personal del nivel de bienestar alcanzado a través de la combinación de las condiciones de vida (variables objetivas que se pueden medir independientemente de los sentimientos) y el grado de satisfacción con la vida (variables subjetivas que dependen de los sentimientos y sólo se pueden mirar a través de los interesados).

También se entendió por adulto mayor a toda persona de 65 o más años, asumiendo que esta población se encuentra en la culminación de un proceso natural con deficiencias funcionales como resultado de cambios biológicos, psicológicos y sociales, condicionado por un fondo genético, los estilos de vida adoptados en la niñez, la acumulación de excesos en la juventud y los aspectos ambientales en que vive el individuo.

La situación actual plantea retos y preocupaciones de vital importancia, tanto para los que los viven como para los organismos gubernamentales, lo que motiva el cuestionamiento de cuáles son las condiciones de vida y de salud de la población adulta mayor. En esta investigación se describen algunas características de la calidad de vida: económicas, familiares, sociodemográficas, de seguridad social y de condiciones de salud de la población adulta mayor de Medellín con el objeto de orientar la implementación de programas de promoción de la salud, prevención, atención y socialización del adulto mayor.

\section{Materiales y métodos}

Se desarrolló un estudio de tipo descriptivo, el cual abordó algunas de las características y condiciones que conforman la calidad de vida de la población adulta mayor de la ciudad de Medellín. Para cada componente se contó con poblaciones y fuentes diversas, complementando estos aspectos con información obtenida a través de una encuesta telefónica realizada en el año 2002, y desglosados como se describe a continuación.

\section{Componentes demográfico y económico}

Se utilizó la información de la Encuesta de Calidad de Vida (ECV) realizada por el Departamento
Administrativo de Planeación Municipal de Medellín, en la cual se usó un diseño muestral por conglomerados polietápico con representatividad por comuna en los años 1997 (4.832 adultos mayores equivalentes a $6,5 \%$ de los 74.869 encuestados) y 2001 (7.296 adultos mayores equivalentes a $7,5 \%$ de los 99.230 encuestados) (7).

\section{Condiciones de salud}

Para el análisis de la mortalidad se utilizaron los certificados de defunción del Departamento Administrativo Nacional de Estadísticas (DANE) de la década de 1990 a 1999 (56.560 adultos mayores fallecidos, equivalentes a $34,9 \%$ de 162.050 defunciones registradas en la ciudad) (8); para la morbilidad por egresos hospitalarios se utilizaron los registros del Ministerio de Protección Social de Colombia de los años 1990 a 1997 (177.121 egresos de adultos mayores equivalentes a $12 \%$ de 1.480 .405 total de egresos hospitalarios) (9), y para la morbilidad sentida se encuestó telefónicamente a 637 adultos mayores.

\section{Componentes social y de seguridad social}

De una muestra estimada de 549, incrementada a 659 adultos mayores de la ciudad, se obtuvo información telefónica de 637, los cuales fueron seleccionados por muestreo sistemático aleatorio de los 6.498 registrados en la ECV del 2001 con número telefónico en sus viviendas (7), basados en que la cobertura del servicio telefónico en la ciudad de Medellín es del 98\%. Para su cálculo se estimó un $95 \%$ de confianza, $4 \%$ de máximo error de muestreo, $50 \%$ de prevalencia del evento en la población y $20 \%$ de incremento, previendo dificultades en la recolección de la información; al término del proceso se obtuvo respuesta del $96,7 \%$ de la muestra incrementada y, por lo tanto, una ausencia de respuesta del $3,3 \%$.

La prueba piloto se realizó telefónicamente a 30 adultos mayores, lo cual permitió efectuar algunos ajustes al instrumento y a la técnica de recolección. La encuesta telefónica fue aplicada por tres encuestadoras contratadas por la Universidad de Antioquia, previamente capacitadas y estandarizadas; en ella se indagó con preguntas cerradas por aspectos demográficos: personas con 
las que vive, número de hijos, ocupación u oficio y condiciones de la vivienda; percepción de condiciones económicas, relacionadas con fuente de ingresos, condiciones económicas del hogar y sentimiento frente a la actual situación económica; sobre seguridad social, en torno al tipo de afiliación al Sistema General de Seguridad Social en Salud, a través del cual se brindan los servicios de salud, y afiliación al fondo de pensiones; sobre las condiciones de salud se indagó por el estado de salud, patologías sufridas, tratamiento de control; sobre las redes de apoyo social (familiar y social) se pidió la opinión sobre las relaciones con familiares y amigos y el apoyo moral recibido, al igual que sobre la capacidad para realizar las actividades de la vida diaria como indicador del grado de independencia percibido.

Los criterios de inclusión contemplaban personas con 65 o más años de edad que tuvieran capacidad mental y sensorial para responder telefónicamente las preguntas y que estuvieran registrados en la ECV; se excluyeron los adultos mayores con trastornos mentales, institucionalizados o en centros geriátricos, puesto que la ECV se realizó sólo en hogares y encuestó a adultos que pudieran responder, excluyendo a aquéllos con limitaciones mentales.

El análisis de la información fue univariado, bivariado y multivariado en cada uno de los componentes de calidad de vida, por cuanto se utilizaron diferentes fuentes de información. Se hizo análisis univariado a las variables sociodemográficas: sexo, edad, ocupación, nivel educativo, condiciones de la vivienda, personas con las que permanece el mayor tiempo; además de la percepción sobre el estado de salud, satisfacción con las redes de apoyo, tanto familiares como sociales, que brindan el apoyo moral, nivel de dependencia y autonomía para realizar las actividades básicas de la vida diaria según la capacidad funcional percibida. El análisis bivariado buscó asociación estadística entre algunas variables sociodemográficas y las del componente específico, por medio de las pruebas ji cuadrado de asociación y de $U$ de Mann Whitney.

Para el análisis multivariado se utilizó el análisis factorial con el método de componentes principales de datos categóricos. Para ello se hizo cuantificación óptima (Optimal Scaling) (Castaño E. Cuantificación de variables cualitativas y análisis de componentes principales. Memorias Simposio de Estadística: análisis Multivariado de Datos. Bogotá: Universidad Nacional de Colombia;1994), y se empleó el procedimiento PRINQUAL (Castaño E. Simposio de Estadística: análisis multivariado de datos realizado en la Universidad Nacional de Colombia, Bogotá, 1994); se determinó dentro de cada componente las variables que mejor explicaban o más cargaban cada uno de ellos, tomando como criterio de selección las variables que aportaran más del $60 \%$ a la variabilidad del factor seleccionado.

\section{Resultados \\ Componente demográfico}

La tendencia demográfica que se registra en la ciudad de Medellín es similar a las tendencias mundiales, pero el índice de envejecimiento en cada país tiene un comportamiento único determinado por el momento, la magnitud y la velocidad del envejecimiento de la población.

Medellín registraba en el censo nacional de 1964 una población adulta mayor de 65 años y más equivalente al 3,4\% (26.271) de la población total; durante 1985 la población de este grupo de edad era de $3,53 \%$ del total de la población, pero a partir de esta fecha, la población anciana aumentó aceleradamente con respecto a otros grupos de edad. Para junio de 2001 se calculó esta población en 105.612, equivalente al $5,7 \%$, sólo en el área urbana y según las proyecciones de la Subdirección de Metroinformación, Departamento Administrativo de Planeación de la ciudad de Medellín (10) (figura 1).

En 1964 la población de Medellín presentaba 87 hombres por cada 100 mujeres y en los adultos mayores había 62 hombres por cada 100 mujeres; para el año 2001 había 85 hombres por cada 100 mujeres en la población total de la ciudad y en adultos mayores el índice era de 58 hombres por cada 100 mujeres.

Según la ECV realizada en la ciudad entre 1997 y 2001, el estado civil de mayor proporción de los adultos mayores encuestados en los dos años 


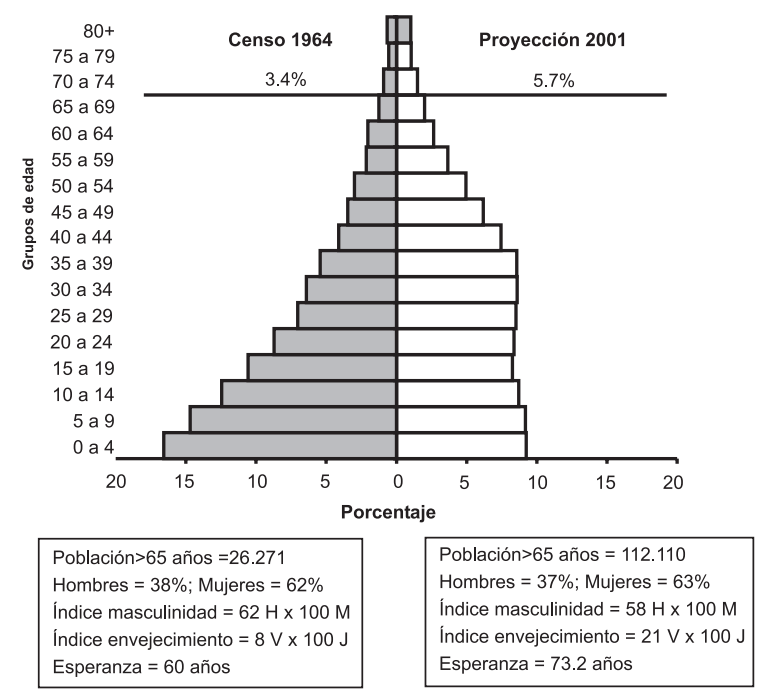

Fuentes: DANE Censos de Población y Vivienda 1964 y 1993.

Departamento Administrativo de Planeación Municipal, Proyección Poblacional 2001

Figura 1. Población total de Medellín para los años 1964 y 2001.

de estudio era el de casados con 46,9\% (2.264) y $45,5 \%$ (3.365), respectivamente, seguido de los viudos, cuya proporción no varía, con 34\% (1.642) y $34,4 \%$ (2.544); pero la condición civil de más variación es la de separados, al pasar de 3,4\% a $5,9 \%$. El estado civil predominante en el hombre es el casado mientras en las mujeres el estado civil más común encontrado es el de viudas y, en menor proporción, casadas, resultado esperado por la alta mortalidad de la población masculina.

Al analizar el nivel de escolaridad alcanzado por los adultos mayores participantes en la ECV, se halló que en 1997 el 52,9\% (2.551) había aprobado la primaria y el $24,3 \%$ (1.174), la secundaria. Para el año 2001, las personas en proceso de envejecimiento con primaria como máximo grado de escolaridad habían aumentado al $63,9 \%$ (4.726) y los que tenían estudios de secundaria bajaron al 19,2\% (1.419). En el 2001 se evidenció un leve incremento en los estudios de posgrado alcanzado por los adultos mayores, pues el $0,1 \%$ manifestó haber alcanzado este nivel (8).

\section{Componente económico}

El ingreso mensual promedio de los adultos mayores en la ciudad de Medellín pasó de
$\$ 182.510$, equivalentes a 1,06 salarios mínimos legales vigentes (SMLV) de 1997 a \$196.537, equivalentes a 0,69 SMLV de 2001; es decir, los adultos mayores tuvieron una pérdida en sus ingresos de $65 \%$ entre 1997 y 2001 . También se observaron diferencias cuando se analizó el ingreso mensual según sexo: en el año 1997 el 28\% (372) de los hombres no recibía ningún ingreso frente al $57 \%$ (889) de las mujeres, y para el 2001, el 30\% (926) de los hombres adultos mayores no recibía ingresos mensuales, recibiendo en promedio $\$ 281.901$ (0,99 SMLV); en este mismo año, el 58\% (2478) de las mujeres no recibían ingresos y en promedio recibían $\$ 134.836(0,47$ SMLV).

Según la encuesta telefónica, se encontró que la principal ocupación u oficio era la de ama de casa en un $44,1 \%$ (281), seguida por los pensionados/ jubilados en un $32,3 \%$ (206). Se halló que el 16,8\% (107) estaba desempleado y sólo el 6,8\% (43) era empleado u obrero.

\section{Condiciones de salud}

\section{Mortalidad}

Las 56.560 muertes registradas en la ciudad de Medellín durante los años 1990-1999 en la población adulta mayor muestran que por cada mil personas adultas mayores que vivían en la ciudad durante este período, fallecieron 57 de ellas en promedio, distribuidas proporcionalmente por sexo en $53,2 \%$ (30.062) del femenino y $46,9 \%$ (26.498) del masculino. La tasa promedio de mortalidad en los hombres fue de 60 por cada mil adultos mayores y en las mujeres de 55 por cada mil.

El lugar de defunción predominante en el adulto mayor es la casa o domicilio con un $48 \%$ (27.126), y en segundo lugar, instituciones de salud como hospitales, clínicas, puestos o centros de salud, con un $43 \%$ (24.115); entre las mujeres el sitio de defunción más común fue la casa, 51,3\% (15.410), mientras que en los hombres su deceso se registró en alguna institución de salud, 45,2\% (11.978).

Al analizar la tasa de defunción por 1.000 habitantes de los diferentes grupos de causas por sexo, se encontró que la mayor razón de 
mortalidad corresponde a accidente de tránsito, y que los hombres tienen 2,9 veces más riesgo de morir por esta causa que las mujeres; le siguen, en su orden, las defunciones por tumor maligno de los órganos genitourinarios, presentándose en los hombres un riesgo de 1,8; por tumor maligno de órganos respiratorios e intratorácicos, los hombres tienen 1,6 veces más riesgo que las mujeres. En el caso de las mujeres, éstas tienen 4,9 veces más riesgo de morir por tumor maligno de los huesos, del tejido conjuntivo o de la piel que los hombres; por enfermedad de las glándulas endocrinas, del metabolismo y trastornos de la inmunidad tienen un riesgo de 1,4 veces más, y por enfermedad hipertensiva el riesgo es de 1,3 veces frente a los hombres que fallecen por la misma causa (figura $2 \mathrm{~A}$ ).

En términos generales, la mortalidad por infarto agudo del miocardio es la principal causa de muerte en la población adulta mayor $(9,5 \%)$, pero a pesar de ello, la obstrucción crónica de las vías respiratorias $(4,5 \%)$, el tumor maligno de bronquio y pulmón $(2,2 \%)$ y la diabetes mellitus $(1,7 \%)$ vienen incrementando sus tasas de mortalidad; la bronconeumonía (1,5\%) permanece estable con tendencia a la baja, a pesar de ocupar los primeros lugares al inicio de la década estudiada.

\section{Morbilidad por egreso hospitalario}

De los egresos hospitalarios de la población total, el $6,1 \%$ se debe a hiperplasia prostática, la cual se presentó en 110 de cada 1.000 adultos mayores de la población; así mismo, del total de egresos registrados por los adultos mayores, la hiperplasia prostática aportó el $60,5 \%$, situación explicable en esta patología cuyas manifestaciones clínicas se inician a alrededor de los 45 años.

La obstrucción crónica de las vías respiratorias representa el 5,6\% de los egresos en los adultos mayores; por cada 1.000 adultos mayores, 100 sufrieron dicha patología. Asimismo, del total de egresos de la población por esta patología, el $67,5 \%$ de ellos corresponde a la población adulta mayor.

Al discriminar por sexo los egresos hospitalarios, se encontró que los hombres presentaron un mayor riesgo de padecer infarto agudo del miocardio y otras cardiopatías congénitas. Las

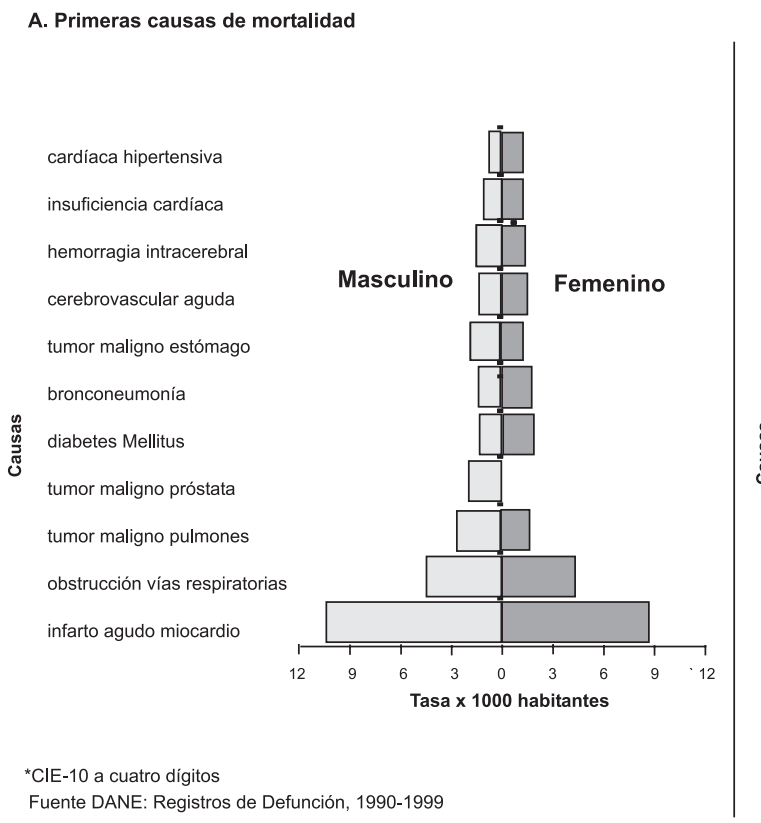

B. Primeras causas de morbilidad por egreso hospitalario

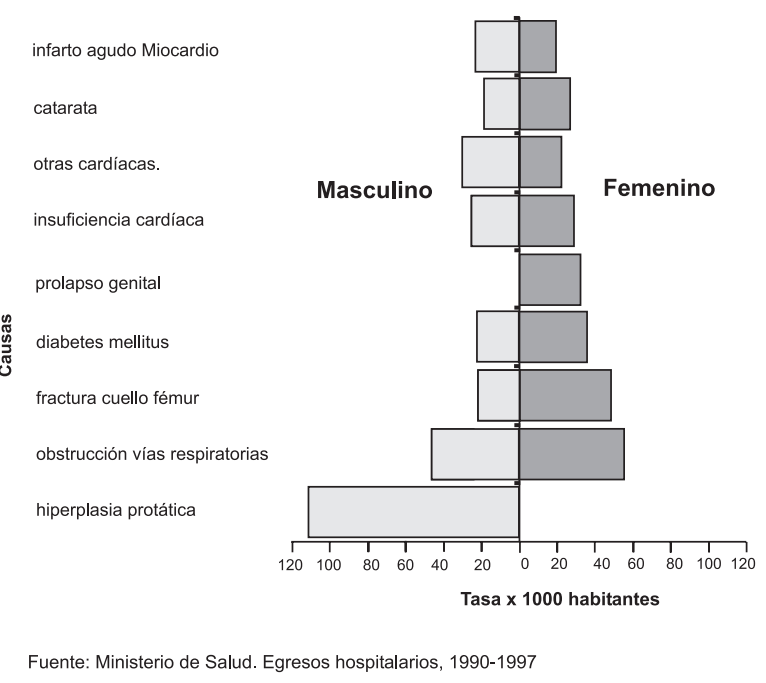

Figura 2. Primeras causas de mortalidad y de morbilidad por egreso hospitalario en la población adulta mayor por sexo. Medellín, 1990-1999. 
mujeres registraron una mayor proporción en patologías como la fractura del cuello del fémur, la diabetes mellitus, la colelitiasis, la catarata y la bronconeumonía (figura 2B).

\section{Morbilidad sentida}

Los adultos mayores manifiestan padecer patologías en uno o varios sistemas simultáneamente; las enfermedades del sistema cardiovascular se presentan en la mitad de la población encuestada $(51 \%)$, siendo la patología más común la hipertensión, presente en $38 \%$ de ellos. Uno de cada cinco adultos mayores encuestados presenta patologías del tejido osteomuscular y conjuntivo (21\%), y en igual proporción padecen del sistema endocrino y metabólico (21\%).

\section{Componente de seguridad social}

Según la ECV, en cuanto a la afiliación al Sistema General de Seguridad Social en Salud (SGSSS) de los adultos mayores, en 1997 el 61,4\% (2.876) se encontraba en el régimen contributivo como contribuyente directo o como beneficiario; en el régimen subsidiado se encontraba el 15,6\% (731) y como vinculado al sistema se encontraba el 23,1\% (1.081); para el año 2001 se conservaban las condiciones de afiliación al régimen contributivo en $64,7 \%$ (4.786), pero la afiliación al régimen subsidiado desciendió a 6,6\% (485) y aumentó la no afiliación a 28,7\% (2.125).

El análisis por sexo de la afiliación al SGSSS mostró que los hombres conservan su afiliación principalmente al régimen contributivo en los dos años estudiados (51,4\% y 52,4\%), pero aumenta la no afiliación del 20,5\% (396) al 25,7\% (799), y el estatus de beneficiario pasa del $12,2 \%$ (236) al $16,3 \%$ (506); la afiliación al régimen subsidiado baja del 15,9\% (307) al 5,5\% (172). En el caso de las mujeres, la afiliación es principalmente como beneficiarias o dependientes, pasando del $29,7 \%$ (817) al 38,2\% (1.641), pero aumenta la no afiliación del 24,9\% (685) al 30,9\% (1.326) y disminuye su participación como contribuyentes directas del régimen contributivo, pasando del $30,1 \%(829)$ al $23,6 \%(1.013)$ y del régimen subsidiado, bajando del 15,4\% (424) al 7,3\% (313).

Según la encuesta telefónica, una alta proporción (79\%) de los adultos mayores encuestados no cuenta con fondo de pensiones; el 21\% (134) restante está afiliado, y muchos de ellos ya disfrutan de una pensión de jubilación en cumplimiento de las normas legales, por enfermedad o por ser cónyuge sobreviviente de un pensionado.

\section{Componente social}

Según el 42,9\% (273) de los adultos mayores, las decisiones importantes y trascendentales que se toman en el hogar donde habita cuentan siempre con su participación, opinión y respeto; ocasionalmente solicitan su opinión o requieren de su participación en un 22,3\% (142) de las veces; esporádicamente o sólo a veces permiten o requieren de su presencia en un 19,2\% (122) y nunca lo hacen en un $9,3 \%$ (59). Se evidencia cómo en el $57 \%$ de las oportunidades, los ancianos no son invitados a participar como un integrante más de la familia en las decisiones fundamentales del hogar.

Consultados por los aspectos de más preocupación, tensión, estrés, depresión o ansiedad, un $35,5 \%$ (226) se refirió a los asuntos relacionados con la economía; un 22,3\% (142) a los asuntos familiares y un $19 \%$ (121) a los relacionados con la salud. Estos tres aspectos (económicos, familiares y de salud) ocupan, en su orden, el $77 \%$ de las preocupaciones de los adultos mayores encuestados.

Cuando un adulto mayor se siente triste, busca el apoyo moral en los más allegados. La tercera parte de los encuestados encuentra ayuda en su familia $(34,6 \%)$; en la oración encuentra consuelo la cuarta parte (25,6\%); en los amigos y vecinos, el $8,8 \%$, y en la música, el $7,9 \%$. Llamó la atención que el $8,7 \%$ no se siente triste ni deprimido, y el $12,4 \%$ utiliza otra forma de apoyo moral para salir del estado depresivo en que se encuentra.

La independencia se indagó a través de la capacidad funcional percibida para realizar las actividades básicas de la vida diaria, lo que permite llevar una vida independiente y autónoma. En general, el $71,6 \%$ (456) se consideró independiente, el 22\% (140). parcialmente dependiente y el 6,4\% (41), dependiente; pero al indagar entre los 526 adultos mayores que 
respondieron directamente la encuesta (o sea, respondieron ellos y no sus cuidadores), el $77,4 \%$ (407) se consideró independiente, el 19,9\% (105) parcialmente dependiente y dependiente sólo el $2,7 \%(14)$.

Las actividades básicas cotidianas de carácter físico, como tomar los alimentos, utilizar correctamente los servicios sanitarios, manejar la incontinencia, trasladarse dentro del hogar, cuidar de la higiene personal y el autocuidado de su persona y seleccionar y ponerse los vestidos que desea, son realizadas por $96 \%$ de los adultos mayores sin muchas dificultades.

El $80 \%$ realiza las actividades de más complejidad, o sea las instrumentales que resultan de la interacción de factores físicos y psicológicos como salir a la calle, usar transporte, tomar medicamentos, hacer su propia comida, hacer trabajo liviano y pesado en casa, cortarse las uñas, usar el teléfono, manejar dinero, abrir y cerrar ventanas, encender y apagar la radio y la televisión. Las actividades más difíciles de realizar son: salir solo a la calle para $71,6 \%$ (456); tomar un medio de transporte (bus, metro o taxi) para $61,5 \%$ (392), y hacer sus propias compras para $72,1 \%(459)$.

El $90 \%$ de los encuestados se consideró en capacidad funcional de realizar actividades que implican contacto social e interrelación con las demás personas y aquéllas que resultan de la interacción de factores físicos, psicológicos, sociales y ambientales como encender la televisión, la radio, leer una revista o un periódico y estar en capacidad mental de entender lo que allí se dice.

Respecto a las ayudas ortopédicas o mecánicas que requiere el adulto mayor para la realización de sus actividades básicas cotidianas 0 esporádicas, se encontró que el $68 \%$ de los encuestados utilizan anteojos y el $10 \%$ utilizan la ayuda del bastón. Otras ayudas ortopédicas son el caminador, la silla de ruedas y las muletas, todas ellas para facilitar el desplazamiento y brindar algún grado de independencia en las actividades básicas cotidianas. El $26 \%$ de los adultos mayores encuestados no utiliza ninguna ayuda.

\section{Análisis de componentes principales: calidad de vida}

Los componentes que mejor explican la calidad de vida de los adultos mayores de la ciudad de Medellín son la independencia, la economía y la seguridad social y las redes de apoyo social; estos tres explican en un $97,4 \%$ (según el alfa de Cronbach) la variabilidad total de la calidad de vida de los adultos mayores. De esta forma se encontró que las variables relacionadas con la independencia explican en un $91,7 \%$ el comportamiento de este componente; las variables económicas y de seguridad social explican en un $83,9 \%$ dicho componente, y las variables de las redes de apoyo social lo explican en un $79,4 \%$. Las variables objetivas referentes a las condiciones materiales de la vivienda del adulto mayor fueron excluidas por el modelo.

\section{Discusión}

Al igual que en muchas regiones del mundo, el envejecimiento de la población de Medellín tiene un mayor peso de mujeres, las cuales presentan una mayor longevidad (58\% de los adultos mayores son mujeres). Esta situación corrobora lo afirmado por la Organización Panamericana de la Salud, la cual enmarca la feminización del envejecimiento como un desafío, por cuanto en casi todos los países, las mujeres sobreviven más tiempo que los hombres, mostrando así que los problemas de las mujeres son los problemas del adulto mayor $(11,12)$.

Tanto en los componentes sociodemográficos como económicos, uno de los aspectos más relevantes es el de las relaciones de género entre los grupos que envejecen, ya que son las mujeres las víctimas de las desigualdades del desarrollo, muchas de ellas son viudas que dependen totalmente de los sistemas de pensión y de los arreglos familiares, siendo evidente el hecho de tener un nivel educativo más bajo (12). Así, estructuralmente están condenadas a vivir más tiempo en condiciones de suma pobreza (13), a ser más tiempo amas de casa, a permanecer en ella y a fallecer en ella, siendo éste el primer sitio de defunción de la población adulta mayor femenina (51\%). 
Así mismo, las personas que en estos días llegan a la edad de jubilarse quieren envejecer con éxito. Este fenómeno es nuevo con relación a las generaciones anteriores: cada vez más sujetos están a sus 65 años en muy buen estado de salud y desean mantenerlo, lo cual es comprensible porque su esperanza de vida promedio a tal edad es de más de 20 años en algunas regiones del mundo (14); para Medellín, la esperanza de vida a los 65 años es de 14,3 años, siendo para los hombres de 13,7 y para las mujeres de 14,9 años.

Otro aspecto importante que rodea la vida del adulto mayor es su situación económica, situación que afecta directamente a las personas adultas mayores, pues al ser catalogadas como sujetas al envejecimiento laboral, se las considera incapaces de trabajar y ser productivas para la sociedad, perdiendo la posibilidad de aportar a los ingresos familiares y convirtiéndose en ocasiones en dependientes (15). Es así como en Medellín, el ingreso mensual de los adultos mayores sufrió un deterioro, ya que pasaron de percibir un 1,06 SMLV en 1997 a un 0,69 SMLV en el año 2001. Esta pérdida en los ingresos fue de $65 \%$ entre 1997 y 2001, deterioro en los ingresos que se observa en otros países de Latinoamérica, como es el caso de Barbados, donde se encontró que la mediana del ingreso per cápita en los adultos mayores (US\$ 3.132) es mucho menor que en el grueso de la población (US\$9.750) (16).

En lo relacionado con la seguridad social, en Colombia las personas más pobres están más desprotegidas que las menos pobres (17); así mismo, los adultos mayores registran desprotección del SGSSS, encontrándose que en Medellín el $65 \%$ está afiliado al régimen contributivo como contribuyente directo o como beneficiario. Este resultado evidencia el aumento porcentual de personas adultas mayores no afiliadas al sistema, por cuanto muestra que las condiciones económicas y de salud influyen directamente en la atención preventiva, tratamiento oportuno y rehabilitación necesarios en los estados mórbidos y mortales de este grupo poblacional.

En los aspectos familiares, el $57 \%$ de los adultos mayores encuestados afirmaron no tener una participación activa en la toma de decisiones del hogar, factor o desarmonía que se evidenció de igual forma en dos investigaciones realizadas en la ciudad de la Habana, Cuba $(18,19)$. Esta situación es el reflejo del "rol sin rol" que ocupa el adulto mayor en su hogar, al no ser invitado a participar como un integrante más de la familia en la toma de decisiones fundamentales en el hogar, llevándolo a perder ese sentido de control que debe tener el adulto mayor sobre aspectos relacionados con su modo de vivir (15). Esta situación muestra el aislamiento del adulto mayor de su vínculo familiar, lo que lo lleva a retraerse y a deprimirse, ocasionándole sentimientos de pérdida, tristeza, desinterés por las actividades, merma de la energía, pérdida de la confianza y autoestima, sentimiento injustificado de culpabilidad e ideas de muerte y suicidio (20).

Por otro lado, aunque la actual generación de adultos mayores sea más proclive a mantenerse vigente y autónoma, y se interese en adquirir nuevos conocimientos para así tener una vejez mejor en términos de calidad de vida, la pobreza intelectual puede ser tan limitante como la material, por las exigencias de la sociedad que aumenta la segregación de los que están en peores condiciones (21); en esta situación se encuentran gran parte de los adultos mayores de la ciudad, donde en el año 2001 se encontró que el $63,9 \%$ sólo contaba con estudios aprobados de primaria y el $19,2 \%$ de secundaria.

En forma general, la investigación estableció que los componentes que explican de mejor manera la calidad de vida del adulto mayor en la ciudad de Medellín son la independencia, la economía y la seguridad social y las redes de apoyo social, explicando éstas tres un $97 \%$ de la variabilidad total de la calidad de vida; estos aspectos son ante todo subjetivos, dejando de lado aquellos relacionados con las tenencias materiales, lo que no significa que tengan poca importancia (aspectos objetivos); de todas maneras priman aspectos como la autonomía, la independencia, la seguridad social y la percepción económica, la percepción del estado de salud y las redes de apoyo social (aspectos subjetivos). Este resultado concuerda con lo planteado por lan Darnton Hill (22), quien considera que los esfuerzos para atender las necesidades de las personas de edad 
se centran en tres factores: seguridad socioeconómica, bienestar psicosocial y sensación de salud.

\section{Conflicto de intereses}

Los investigadores declaramos que durante la realización del proyecto de investigación no existió conflicto de intereses entre la entidad financiadora (Universidad de Antioquia), las entidades que cedieron la información (Municipio de Medellín y Departamento Administrativo Nacional de Estadísticas), los investigadores ni los encuestados, que pudieran haber afectado los resultados del mismo.

\section{Financiación}

La entidad financiadora fue la Facultad Nacional de Salud Pública de la Universidad de Antioquia.

\section{Referencias}

1. Villanueva LA. Sobre el envejecimiento: una perspectiva integral. Revista Hospital General Doctor Manuel Gea González 2000;3:107-14.

2. Organización Panamericana de la Salud. Tendencias demográficas y de mortalidad en la Región de las Américas, 1980-2000. Boletín Epidemiológico 2002;23:1-4.

3. Organización Panamericana de la Salud. División de Promoción y Protección de la Salud. Salud de las personas de edad. Envejecimiento y salud: un cambio de paradigma. Rev Panam Salud Publica 2000;7:60-7.

4. Rueda S. Habitabilidad y calidad de Vida (en línea). 1997 [Consultado 2001 may 25]. Disponible en: http:// habitat.aq.upm.es/cs/p2/a005.html.

5. Organización Mundial de la Salud. Programa Envejecimiento y Ciclo Vital. Envejecimiento activo: un marco político. Rev Esp Geriatr Gerontol 2002;37:74105.

6. Fleury E, Lana da Costa C. Qualidade de vida e saúde: aspectos conceituais e metodológicos. Cad Saúde Pública 2004;20:580-8.

7. Departamento Administrativo de Planeación Municipal. Encuesta Calidad de Vida 1997 y 2001. Medellín: Alcaldía, Subdirección de Metroinformación; 2002.

8. Departamento Administrativo Nacional de Estadística. Certificados de defunción 1990-1999. Bogotá: DANE; 2002.
9. Ministerio de Salud. Registros de egresos hospitalarios 1990-1997. Bogotá: Instituto Nacional de Salud; 2001.

10. Departamento Administrativo de Planeación Municipal. Distribución del número de personas encuestadas y clasificadas por el SISBEN, según grupos de edad y comuna de residencia habitual. Medellín: Alcaldía, Subdirección de Metroinformación; 2000.

11. Organización Mundial de la Salud. Departamento de Promoción de la Salud. Salud y Envejecimiento. Un documento para el debate. Segunda Asamblea Mundial. Madrid: OMS; 2002

12. Albala C, Lebrao ML, León Díaz EM, Ham-Chande R, Hennis AJ, Palloni A, et al. Encuesta Salud, Bienestar y Envejecimiento (SABE): metodología de la encuesta y perfil de la población estudiada. Rev Panam Salud Pública 2005;17:307-22.

13. Montes de Oca V. Envejecimiento y modernidad: impactos demográficos. Nueva Sociedad 1994; 129:132-41.

14. Pierre V. Envejecer exitosamente: concebir el proceso de envejecimiento con una perspectiva mas positiva Salud Pública Mex 1996;38:513-22.

15. Barros C. Aspectos sociales del envejecimiento. En: Anzola E, Galinsky D, Morales F, Salas A, Sánchez M, editores. La atención de los ancianos: Un desafío para los años noventa. Publicación científica 546. Washington D.C.: OPS; 1994. p.57-66.

16. Hambleton IR, Clarke K, Broome HL, Fraser HS, Brathwaite F, Hennis AJ. Historical and current predictors of self-reported health status among elderly persons in Barbados. Rev Panam Salud Publica 2005;17:342-52.

17. López H. La vejez urbana en Colombia. Bogotá: Fundación Friedrich Ebert de Colombia, Fescol; 1993.

18. Fernández S, Almuiña M, Chil OA, Blanco B. Factores psicosociales presentes en la tercera edad. Rev Cubana Hig Epidemiol 2001;39:77-81.

19. Couse C, Zamora M, Purón I, del Pinto IA. La bioética y los problemas del adulto mayor. Medisan 1998;2:30-5.

20. Organización Mundial de la Salud. Informe sobre la salud en el mundo. Salud mental: nuevos conocimientos, nuevas esperanzas. Ginebra: OMS; 2001.

21. Domínguez 0 . Encuesta de necesidades de los ancianos en Chile. Percepciones de los adultos mayores sobre sí mismos (en línea). 1984. [Consultado enero 10 de 2001]. Disponible en: http:// www.gerontologia.uchile.cl/docs/chien5.htm.

22. Darnton-Hill I. El envejecimiento con salud y la calidad de vida. Foro Mundial de la Salud 1995;16:381-91. 